\title{
ENVELHECIMENTO E HIVIAIDS NA PARAÍBA: ANÁLISE EPIDEMIOLÓGICA, 2009-2018
}

\author{
AGING AND HIVIAIDS IN PARAÍBA: EPIDEMIOLOGICAL ANALYSIS, \\ 2009-2018
}

\author{
Ariadne Pereira Pedroza ${ }^{1}$ \\ Francisco Ronner Andrade da Silva ${ }^{2}$ \\ Rayrla Cristina de Abreu Temoteo ${ }^{3}$ \\ Amanda Haissa Barros Henriques ${ }^{4}$
}

\begin{abstract}
RESUMO: Considerando que aproximadamente a partir 50 anos de idade, surgem alterações biológicas e psicológicas que caracterizam a menopausa na mulher e a andropausa no homem, sinalizando o início do processo de envelhecimento, esse grupo etário chama a atenção dos diversos segmentos sociais pelas importantes mudanças enfrentadas associadas à infecção pelo Vírus da Imunodeficiência Humana (HIV). Objetivo: analisar os dados epidemiológicos do HIVIAIDS na faixa etária em discussão. Método: trata-se de um estudo transversal, retrospectivo, descritivo e de abordagem quanti-qualitativa, realizado no período de agosto a setembro de 2019, sendo os dados obtidos através de consulta a partir das informações disponíveis nos sites TABNET/DATASUS e Portal da Saúde do Ministério da Saúde, através do Sistema de Informação de Agravos de Notificação SINAN, com amostra entre 2009 a 2018. A análise dos dados foi realizada através de estatística descritiva simples, além de análise reflexiva, tendo como embasamento teórico material publicado pertinente ao tema proposto. Resultados: observou-se avanço do número de casos de HIV em pessoas com idade igual ou superior a 50 anos no Brasil, diante da notificação de 27.799 casos durante o período de 2009 a 2018, predominância do sexo masculino, correspondendo a terceira maior incidência, por faixa etária no país. Conclusão: a epidemia do HIV e da AIDS no Brasil não está mais restrita a grupos específicos, tendo sua capacidade
\end{abstract}

\footnotetext{
${ }^{1}$ Enfermeira. Mestre em Enfermagem pela Universidade Federal da Paraíba - UFPB. Docente da Faculdade São Francisco da Paraíba - FASP. Enfermeira Assistencial do Hospital Universitário Júlio Bandeira - HUJB/UFCG/EBSERH. Email: ariadne.pedroza@gmail.com.

${ }^{2}$ Fisioterapeuta. Mestre em Terapia Intensiva - IBRATI. Docente da Faculdade São Francisco da Paraíba - FASP.

${ }^{3}$ Enfermeira. Doutoranda em Enfermagem pela Universidade Federal do Rio Grande do Norte UFRN. Docente EBTT do Instituto Federal da Paraíba - IFPB, campus Mangabeira.

${ }^{4}$ Enfermeira. Mestre em Enfermagem pela UPE/Universidade estadual da Paraíba. Docente EBTT do Instituto Federal da Paraíba - IFPB, campus Mangabeira.
} 
de disseminação em todos os segmentos da sociedade, exigindo maior atenção dos profissionais e serviços de saúde quanto aos aspectos associados ao envelhecimento.

Descritores: HIV e AIDS; Envelhecimento; Sexualidade.

ABSTRACT: Considering that approximately from 50 years of age, biological and psychological changes appear that characterize menopause in women and andropause in men, signaling the beginning of the aging process, this age group draws the attention of the various social segments for the important changes faced associated infection with the Human Immunodeficiency Virus (HIV). Objective: to analyze the epidemiological data of HIV / AIDS in the age group under discussion. Method: this is a cross-sectional, retrospective, descriptive study with a quantitative and qualitative approach, carried out from August to September 2019, with data obtained through consultation based on information available on the TABNET / DATASUS and Portal da Health of the Ministry of Health, through the Information System for Notifiable Diseases - SINAN, with a sample between 2009 and 2018. The data analysis was performed through simple descriptive statistics, in addition to reflective analysis, having as theoretical basis published material pertinent to the proposed theme. Results: there was an increase in the number of HIV cases in people aged 50 years or older in Brazil, in view of the notification of 27,799 cases during the period from 2009 to 2018, predominance of males, corresponding to the third highest incidence, by age group in the country. Conclusion: the HIV and AIDS epidemic in Brazil is no longer restricted to specific groups, having its capacity for dissemination in all segments of society, requiring greater attention from health professionals and services regarding aspects associated with aging.

Keywords: HIV and AIDS; Aging; Sexuality. 


\section{INTRODUÇÃO}

O envelhecimento populacional tem sido considerado como um fenômeno proeminente mundial. A ampliação do tempo de vida está atrelada a uma melhoria substancial dos parâmetros de saúde das populações, tornando para o campo da saúde pública um iminente desafio para lidar com essa mudança. A população brasileira, evidenciada em diversos estudos, vem envelhecendo de forma rápida, sendo caracterizada pela transição demográfica, que indica baixas taxas nos indicadores de fecundidade e mortalidade, além de outros fatores que justificam essa mudança. Independentemente deste fato, entende-se o envelhecimento como fase natural e individual do desenvolvimento humano (MOREIRA et al., 2009).

O aumento do número de pessoas que estão envelhecendo no Brasil, até há pouco tempo considerado um país de jovens, começa a dar lugar a uma realidade diferente e traz a consciência de que a velhice existe e é uma questão social que exige uma atenção diferenciada (ZIMERMAN, 2007).

O envelhecimento populacional vem sendo um dos maiores desafios da saúde pública, principalmente nos países em desenvolvimento. Todavia, envelhecer torna-se uma conquista, visto que, a população brasileira inserida na faixa etária maior ou igual a 50 anos está não apenas vivendo mais, como também melhor. Além disso, os indivíduos que conseguem sobreviver até idades mais avançadas são selecionados por maiores condições de saúde e de qualidade de vida, inclusive prolongando sua atividade sexual (CARBONNI; REPPETTO, 2007).

A Organização Mundial de Saúde (OMS) considera o envelhecimento como um processo natural e individual, que caracteriza a velhice dentre as etapas da vida e define o idoso sendo aquele que se encontra na faixa etária após 60 anos, em países em desenvolvimento como o Brasil, e, acima de 65 anos, nos países desenvolvidos (WHO, 2012).

A elevação da expectativa de vida, associada à melhoria nas condições de saúde e ao avanço da medicina, têm contribuído para aumentar também a 
expressão sexual das pessoas que estão envelhecendo, muitas vezes tidas como pessoas assexuadas (GODOY et al., 2008).

A mudança no comportamento sexual desse grupo etário, o avanço das tecnologias, o acesso a medicamentos para a disfunção erétil, o uso de próteses e à resistência em usar preservativo, conferem um novo perfil epidemiológico da Síndrome da Imunodeficiência Adquirida (AIDS) nos últimos anos (GOMES; SILVA, 2008).

Considerando que aproximadamente a partir 50 anos de idade, surgem alterações biológicas e psicológicas que caracterizam a menopausa na mulher e a andropausa no homem, sinalizando o início do processo de envelhecimento (ROHDEN, 2011), esse grupo etário chama a atenção dos diversos segmentos sociais pelas importantes mudanças enfrentadas por esses indivíduos nesse processo e associado à infecção pelo Vírus da Imunodeficiência Humana (HIV). Nesse contexto que o HIV tem se manifestado nessa faixa etária, a literatura tem denominado esse grupo como idoso (UNAIDS, 2006).

Sendo assim, as pessoas com idade acima de 50 anos, quando infectadas com o HIV, tendem a manifestar os efeitos da imunodepressão de forma mais acelerada que as pessoas jovens, porque têm acrescido a AIDS, os efeitos de outras doenças que frequentemente aparecem com a aproximação do envelhecimento. Em se tratando de pessoas com 65 anos ou mais, os efeitos da AIDS são ainda mais graves (BRASILEIRO; FREITAS, 2006).

A população idosa no Brasil, no início da epidemia do HIV, praticamente não foi atingida. Nos primeiros cinco anos de sua evolução, apenas quatro casos foram diagnosticados em pessoas com 60 anos ou mais (BRASIL, 2007).

Com relação às pessoas com idade igual ou maior que 50 anos, o primeiro caso de AIDS notificado no Brasil foi registrado no ano de 1982, desde então, a contaminação entre esses indivíduos cresce no país, e, embora em menor porcentagem de casos, quando comparados a outros grupos etários, tem aumentado significativamente (BRASIL, 2018).

Nesse sentido, o avanço de casos em pessoas com idade igual ou superior a 50 anos infectados pelo HIV podem ser distribuídas em duas categorias: uma 
contaminada pelo vírus ao iniciar o processo de envelhecimento e outra, por ter sido contaminado já vivendo esse processo.

Diante dessa realidade, muitas causas são atribuídas ao aumento nos índices de contaminação pelo HIV em pessoas inseridas na faixa etária, a exemplo das mudanças socioculturais, sobretudo na sexualidade, e as inovações na área medicamentosa que contribuíram para uma segurança desses indivíduos nas conquistas amorosas. Outro aspecto pode ser justificado pelo fato dessa geração não ter sido orientada com relação à importância de fazer uso de preservativo como estratégia de prevenção para o vírus, além do aumento significativo na perspectiva e qualidade de vida dos portadores do HIV em resposta ao advento da Terapia Antirretroviral (TARV) (SILVA; PAIVA, 2006).

Desse modo, as pessoas com idade acima de 50 anos, quando infectadas com o HIV, tendem a manifestar os efeitos da imunodepressão de forma mais acelerada que as pessoas jovens, porque têm acrescido a AIDS, os efeitos de outras doenças que frequentemente aparecem com a aproximação do envelhecimento. Em se tratando de pessoas com 65 anos ou mais, os efeitos da AIDS são ainda mais graves (BRASILEIRO; FREITAS, 2006).

Há uma tendência de um elevado crescimento no número de pessoas contaminadas pelo HIV com idade igual ou acima de 50 anos, tornando o envelhecer soropositivo para o vírus, um desafio duplicado para quem enfrenta essa situação (SALDANHA et al., 2008). Frente a cronificação da AIDS e a incidência de casos da doença nas pessoas em processo de envelhecimento, essa realidade requer que seja fortalecida as discussões e compreensões, uma vez que a epidemia ainda impõe desafios para seu controle.

Partindo do pressuposto de que cada vez mais indivíduos estão envelhecendo e se contaminando com o HIV após os 50 anos de idade, esse estudo tem por objetivo analisar os dados epidemiológicos do HIV na faixa etária em discussão no Estado da Paraíba, entre os anos de 2009 a 2018. 


\section{METODOLOGIA}

Trata-se de um estudo transversal, retrospectivo, descritivo e de abordagem quanti- qualitativo realizado no período de agosto a setembro de 2019.

O estudo transversal é definido por pesquisa observacional, que analisam dados coletados ao longo de um período de tempo, e retrospectivo por se tratar de dados notificados entre os anos de 2009 a 2018.

Para alcance dos objetivos propostos, os dados foram obtidos através de consulta a partir das informações disponíveis nos sites TABNET/DATASUS (http://www2.datasus.gov.br/DATASUS/index.php?area=02) e Portal da Saúde (http://portalms.saude.gov.br/) do Ministério da Saúde, através do Sistema de Informação de Agravos de Notificação - SINAN.].

Delimitou-se a amostra ao período de 2009 a 2018, cujo cenário corresponde aos casos de HIV (número e percentual) notificados no SINAN segundo sexo e faixa etária, por ano do diagnóstico. A análise dos dados foi realizada através de estatística descritiva simples, além de análise reflexiva, tendo como embasamento teórico material publicado pertinente ao tema proposto.

Por se tratar de estudo com utilização de banco de dados de domínio público, não foi necessária submissão ao Comitê de ética e Pesquisa. Todavia, foram respeitados todos os preceitos éticos contidos na Resolução 466/12 do Conselho Nacional de Saúde, que aprovam diretrizes e normas regulamentadoras de pesquisas envolvendo seres humanos (BRASIL, 1996).

\section{RESULTADOS}

Atualmente tem tido sua potencialidade de disseminação nos segmentos da sociedade, com destaque para sua incidência em todas as faixas etárias, apontando 
para um crescimento acentuado na população jovem e entre as pessoas com mais de 50 anos (BERTONCINI et al., 2007).

\begin{tabular}{|c|c|c|c|c|c|c|c|c|c|c|c|}
\hline $\begin{array}{c}\text { ANO/FAIXA } \\
\text { ETÁRIA }\end{array}$ & 2009 & 2010 & 2011 & 2012 & 2013 & 2014 & 2015 & 2016 & 2017 & 2018 & TOTAL \\
\hline \multicolumn{12}{|c|}{ MASCULINO } \\
\hline 50 a 54 & 215 & 286 & 377 & 412 & 527 & 916 & 1101 & 1238 & 1353 & 580 & 7005 \\
\hline 55 a 59 & 128 & 170 & 187 & 204 & 329 & 503 & 654 & 794 & 794 & 327 & 4090 \\
\hline $\begin{array}{l}60 \text { ou } \\
\text { mais }\end{array}$ & 110 & 150 & 184 & 199 & 333 & 541 & 716 & 825 & 857 & 393 & 4308 \\
\hline TOTAL & 453 & 606 & 748 & 815 & 1189 & 1960 & 2471 & 2857 & 3004 & 1300 & 15403 \\
\hline \multicolumn{12}{|c|}{ FEMININO } \\
\hline 50 a 54 & 174 & 212 & 215 & 255 & 377 & 599 & 756 & 832 & 778 & 358 & 4556 \\
\hline 55 a 59 & 99 & 123 & 155 & 163 & 259 & 385 & 464 & 522 & 503 & 250 & 2923 \\
\hline $\begin{array}{l}60 \text { ou } \\
\text { mais }\end{array}$ & 89 & 108 & 136 & 151 & 217 & 358 & 445 & 516 & 553 & 2344 & 4917 \\
\hline TOTAL & 362 & 443 & 506 & 569 & 853 & 1342 & 1665 & 1870 & 1834 & 2952 & 12396 \\
\hline
\end{tabular}

Tabela 1 - Casos de HIV notificados no SINAN segundo sexo e faixa etária, por ano do diagnóstico. Brasil, 2009-2018

O avanço do número de casos de HIV em pessoas com idade igual ou superior a 50 anos no Brasil tem demandado um olhar de diversos segmentos sociais e científicos, diante da notificação de 27.799 casos durante o período de 2009 a 2018, sendo 15.403 casos do sexo masculino e 12.396 do sexo feminino, correspondendo a terceira maior incidência, por faixa etária no país (BRASIL, 2018), conforme apontado em Tabela 1.

\section{DISCUSSÃO}

Apesar de descrita três décadas da doença, a AIDS ainda se mantém como tema emergente no âmbito da saúde coletiva. Ao evoluir em número de casos nos 
indivíduos com idade igual ou acima de 50 anos, é considerada um tema atual e relevante por gerar implicações morais, socioculturais e religiosas, e pelo impacto na saúde dessa parcela da população (DELMIRO, 2011).

Outro fato importante refere-se ao advento dos estimulantes eréteis que representou mudanças significativas de comportamento na esfera da sexualidade nos idosos, culminando em maior liberdade sexual e exposição ao risco de contágio de diferentes doenças. Com a prática sexual insegura e desprotegida, o HIV e a AIDS nesses indivíduos trouxe à tona uma gama de aspectos que envolvem implicações de natureza epidemiológica, social, psicológica e socioeconômicas.

Durante a primeira década da epidemia (1980-1990), o HIV esteve associado a grupos específicos, seja pela opção sexual ou envolvimento no uso de drogas. $\mathrm{Na}$ segunda década (1990-2000) passou a apresentar outro padrão epidemiológico, avançando entre os heterossexuais, intensificou a feminização e a transmissão vertical (SILVA; PAIVA, 2006).

$\mathrm{O}$ aumento nos índices de contaminação pelo HIV em pessoas com idade igual ou superior a 50 anos apresentam causas variadas como a resistência na utilização do preservativo, seja por questões educativas, culturais, econômicas, dentre outras, e as inovações na área medicamentosa para a melhora do desempenho sexual e da reposição hormonal, favorecendo o aumento da frequência e à qualidade das relações sexuais (REZENDE et al., 2009).

Observa-se predominância do número de casos no sexo masculino, embora um fator agravante diz respeito às mulheres com mais de 50 anos não utilizarem preservativo nas suas relações pelo fato de já terem uma família constituída, de estarem laqueadas ou de não poderem engravidar pelo avanço da idade, gerando a falsa impressão da inutilidade do preservativo em sua vida sexual (SILVA; PAIVA, 2006). Entretanto, não usar preservativo impõe risco, visto que, as paredes vaginais tornam-se mais finas e ressecadas no climatério e depois da menopausa, favorecendo o surgimento de lesões que abrem caminho para as DSTs.

Desse modo, muitos são as causas responsáveis pelo aumento de casos de AIDS nessa faixa etária. Todavia, diferente do início da epidemia, hoje o portador pode ter qualidade e expectativa de vida, além de buscar a vivência plena de sua sexualidade. 
Antes da década de 90 , havia pouca solução terapêutica para arrefecer os efeitos da ação do HIV no indivíduo. Com isso, os pacientes vivenciavam sua evolução clínica e esperavam a morte, sem expectativas alentadoras de tratamento (SANTOS et al., 2007). Porém, com o avanço das pesquisas farmacológicas surgiu às primeiras tentativas terapêuticas mais eficazes e uma maior esperança para os infectados.

O avanço científico e o advento da terapia antirretroviral proporcionaram significativo aumento na expectativa e qualidade de vida dos indivíduos soropositivos e como uma doença incurável, teve sua realidade alterada por um contexto marcado pela cronificação da doença, acarretando novos desafios para sua compreensão e enfrentamento.

\section{CONSIDERAÇÕES FINAIS}

Pessoas em processo de envelhecimento associado à soropositividade para o HIV vivenciam duplos desafios, ou seja, o enfretamento da doença no seu dia-a-dia com alterações e perdas fisiológicas do envelhecer. Essas transformações contribuem para o indivíduo expressar os mais variados sentimentos, que vão desde a negação da situação até a busca pelo isolamento social.

Faz importante entender que a epidemia do HIV e da AIDS no Brasil não está mais restrita a grupos específicos, tendo sua capacidade de disseminação em todos os segmentos da sociedade. Neste contexto, compreender e abordar questões relacionadas à vida afetivo-sexual das pessoas que estão em processo de envelhecimento com idade igual ou superior a 50 e que convivem com HIV ou a AIDS, requer maior atenção dos profissionais e serviços de saúde em proporcionar atendimento integral, contemplando estratégias preventivas e de enfrentamento da doença.

Além disso, a pessoa no processo de envelhecimento se apresenta como um desafio a ser incorporado pela saúde pública, principalmente no Brasil, uma vez que, 
a atenção à saúde do idoso é uma especialidade em expansão que ainda carece de profissionais qualificados.

Diante do contexto, o presente buscou tornar visível uma das facetas, entre tantas outras, que a epidemia tem provocado em termos de desafios e interesses de demandas científicas e políticas preventivas, considerando que pessoas que estão envelhecendo sendo soropositivos para o HIV ou com AIDS estão se deparando com enfrentamentos diversos e construindo relacionamentos que perpassam a condição sorológica.

\section{REFERÊNCIAS BIBLIOGRÁFICAS}

BRASIL. Secretaria de Vigilância em Saúde - Departamento de Vigilância, Prevenção e Controle das Infecções Sexualmente Transmissíveis, do HIV/Aids e das Hepatites Virais. Boletim Epidemiológico HIVIAIDS 2018. Brasília, v. 49. n. 53, 2018.

Ministério da Saúde. Secretaria de Atenção à Saúde. Departamento de Atenção Básica. Envelhecimento e Saúde da Pessoa Idosa. Brasília (DF); 2007.

Ministério da Saúde. Conselho Nacional de Saúde. Comissão Nacional de Ética em Pesquisa. Resolução No 196 de 10 de outubro de 1996: aprova as diretrizes e normas regulamentadoras de pesquisa envolvendo seres humanos. Brasília (DF); 1996.

BRASILEIRO, M.; FREITAS, M. I. F. Representações sociais sobre AIDS de pessoas acima de 50 anos de idade, infectadas pelo HIV. Rev. Latino-Am. Enfermagem. Ribeirão Preto, v.14, n.5, p. 178-195. set/out. 2006.

BERTONCINI, B. Z. et al. Comportamento Sexual em Adultos Maiores de 50 Anos Infectados pelo HIV. DST - J bras Doenças Sex Transm. Rio de Janeiro, v. 19, n. 2, p. 75- 79, 2007.

CARBONI, R. M.; REPPETTO, M. A. Uma reflexão sobre a assistência à saúde do idoso no Brasil. Rev. Eletr. Enf. v. $9 . \quad \mathrm{N} 1$ p. 251-60. 2007. Disponível em: <http://www.fen.ufg.br/revista/v9/n1/pdf/v9n1a20.pdf>. Acesso em: 09 de Mai de 2012.

DELMIRO, R. S. O que pensam os idosos sobre a AIDS: representações sociais e práticas. 2011. 90f. Dissertação (Mestrado em Enfermagem e Saúde) - Universidade Estadual do Sudoeste da Bahia. Bahia 2011. Disponível em: $<$ <ttp://www.uesb.br/ppgenfsaude/dissertacoes/turma1/Disserta\%C3\%A7\%C3\%A30\%20Rosan a\%20Delmiro.pdf>. Acesso em: 30 Jun 2012.

GODOY, V. S. et al. O perfil epidemiológico da AIDS em idosos utilizando Sistemas de Informações em Saúde do DATASUS: realidades e desafios. DST - J bras Doenças Sex Transm. Rio de Janeiro, v. 20, n. 1, p. 7-11, 2008.

GOMES, S. F.; SILVA, C. M. da. Perfil dos Idosos Infectados pelo HIVIAIDS: uma revisão. Vittalle. Rio Grande, v. 20, n.1, p. 107-122, 2008.

MOREIRA, A. S. P. et al. Percepção dos estudantes sobre o idoso e seus direitos: o caso da saúde. Rev Gaúcha Enferm. Porto Alegre, v. 30. n. 4. p. 654-660. dez. 2009. 
REZENDE, M. C. M. et al. Aids na terceira idade: determinantes biopsicossociais. estudos. Goiânia, v. 36, n. 1/2, p. 235-253. jan./fev. 2009. Disponível em: <http://revistas.ucg.br/ index.php/estudos/ article/viewFile/1027/725>. Acesso em: 12 Jun de 2011.

ROHDEN, F. "O homem é mesmo a sua testosterona": promoção da andropausa e representações sobre sexualidade e envelhecimento no cenário brasileiro. Horiz. antropol. Porto Alegre, v. 17, n. 35. jan./jun. 2011.

SALDANHA, A. A. W. et al. Representações sobre a Aids na velhice por coordenadoras de grupos da terceira idade. Psico-USF. São Paulo, v. 13, n. 1, p. 95-103. jan./jun. 2008. Disponível em: <http://www.scielo.br/pdf/pusf/v13n1/v13n1a12.pdf>. Acesso em: 15 de Jun de 2011.

SANTOS, E. C. M. dos. et al. Qualidade de vida de pessoas vivendo com HIV/Aids em São Paulo. Rev. Saúde Pública. São Paulo, v. 41. supl. 2. p. 64-71. dez. 2007.

SILVA, L. S.; PAIVA, M. S. Vulnerabilidade ao HIVIAIDS entre homens e mulheres com mais de 50 anos. In: VII Congresso Virtual HIVIAIDS - O VIH/SIDA na criança e no idoso. Santarém: Metatexto, 2006. p. 85-104.

UNAIDS. WORLD HEALTH ORGANIZATION. Estigma, discriminação e violação dos direitos humanos em relação ao VIH. 2005. Disponível em: < http://whqlibdoc.who.int/publications/2005/929173344X_por.pdf>. Acesso em: Jan de 2013. ZIMERMAN, G. I. Velhice: aspectos biopsicossociais. Porto alegre: Artmed, 2007. 